\title{
Analysis and Selection of Appropriate Components for Power System Metrology Instruments
}

\author{
Quijano Cetina, R. ${ }^{1}$, Yljon Seferi ${ }^{1}$, Steven M. Blair ${ }^{1}$, Paul S. Wright ${ }^{2}$ \\ ${ }^{1}$ Dept. of Electronic and Electrical Engineering, University of Strathclyde, Glasgow, UK, renan.quijano-cetina@strath.ac.uk \\ ${ }^{2}$ National Physical Laboratory (NPL), Teddington, UK
}

\begin{abstract}
Modern and future power systems require accurate measurements for a wide range of applications such as control, protection, and electrical energy consumption billing. The overall accuracy of measured electrical quantities depends on individual error contributions from different stages of the signal path within the measurement instrument, as well as power quality conditions of the grid. Present developments in hardware and signal processing techniques allow accurate measurements of voltage and current signals in almost any possible scenario. Nevertheless, in order to achieve the required level of accuracy, considerations should be given when choosing components for the different stages of the measuring instrument design. In this paper, a review and analysis of some of the most common hardware components and signal processing techniques is presented. Recommendations are given to provide practical guidance for developing power system measurement devices, which focus on the applications of electrical energy metering and phasor measurement units.
\end{abstract}

Index Terms-Electrical measurements, power quality, analogue front-end, phasor measurement unit.

\section{INTRODUCTION}

Measurements play a key role in modern power systems as they allow system operators to understand the status of the electrical grid. Moreover, accurate and timely measurements enable a fast response from control and protection systems [1]. While sophisticated measurement instruments such as phasor measurement units (PMU) and power quality (PQ) analysers are available on the market, they are not flexible (for the user) and are usually expensive. Therefore, for example, when a particular feature or measurement, which is not performed as required (e.g. greater bandwidth is needed to measure high order harmonics, or RMS must be calculated over a half cycle), is needed to monitor the electrical grid, engineers may need to design a custom-made instrument.

The overall accuracy of any measurement system or instrument is affected by the systematic error contributions of different sub-systems or elements [2]. Therefore, in order to appropriately design an electrical measurement system, it is important to carefully select suitable components for each stage. In the following sections, a brief description of the components and calculation techniques for electrical measuring instruments is presented.

\section{ANALOGUE FRONT END}

The analogue front-end components (Fig. 1) for sampling-based instruments designed to measure electrical quantities are typically similar regardless of the function of the device (e.g. smart electricity meter, power quality analyser, or protection relay). However, depending on the particular application, certain features or characteristics, should be considered for each stage. In this section, guidance on the selection of appropriate components for different applications is provided.

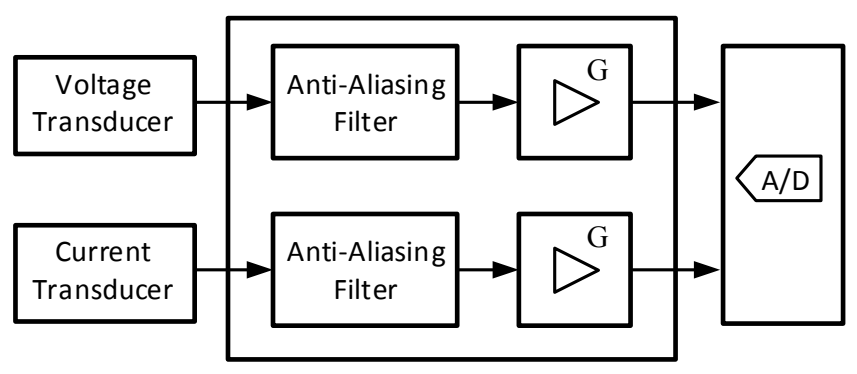

Fig. 1. Electricity Meter Analogue Front End.

\section{A. Transducers}

The most common transducers for the aforementioned measurement applications are: voltage transformers (VT) or resistive voltage divider (RVD), for the voltage inputs; and current transformers (CT), current shunt resistors, Rogowski coils, zero-flux transformers or Hall effect clamps for the current inputs. When choosing a particular transducer (or sensor) technology, it is important to consider not only the accuracy of the transducer but also some characteristics such as frequency response and phase delay, in order to reduce additional sources of error.

Inductive voltage and current transformers are broadly used in measurement systems together with electricity meters, PMUs and power quality analyzers, as they are very accurate. However, these sensors introduce a ratio error and a phase displacement error [3], [4] which is critical for the performance of a PMU; and should be compensated accurately. CTs and VTs frequency response is limited and in general lower compared to the frequency response of a resistive voltage divider [5]. Therefore, for applications that require measurement of high frequencies, such as harmonics or other power quality phenomena which are characterized by frequencies higher than 50 or $60 \mathrm{~Hz}$, resistive voltage dividers might be a better choice compared to inductive transformers. 
The phase response is particularly important for power measurements, and clamps and Hall effect devices can often have phase errors of several degrees. Phase is also important for waveform shape reconstruction, where increasing phase errors at higher frequency harmonics can distort the time domain shape of a captured waveform. If the phase error is stable and can be measured, it is possible to correct the response using deconvolution methods [6].

Openable Rogowski coils and Hall clamps have the advantage of non-invasive measurements without the need to break the current circuit. However, they are susceptible to significant errors due to rotation and position on the current carrying conductor [7]-[9].

The requirement to have a dc-coupled input is also relevant to the choice of the transducer, as power system metrology applications for power quality often need to measure very low frequencies, such as subsynchronous interharmonics. A dc-coupled transducer also achieves a better input match or voltage standing wave ratio (VSWR) performance, compared to an ac-coupled transducer, due to the absence of a large in-series capacitor.

Overload protection circuits are also used in commercial instruments; these may be fused circuits using diode or transient voltage suppression (transorb) circuits. High rupturing capacity (HRC) fuses should be used for substation situations where the fault level is high. Care should be taken that these circuits do not introduce amplitude and phase errors.

\section{B. Anti-Aliasing Filters}

Depending on the analogue to digital converter (ADC) sampling frequency, appropriate anti-aliasing (AA) filters should be placed directly after the transducers. Oversampling ADCs eases the design requirements of the AA filter allowing for low order filters that can be implemented passively. For power and energy applications, the cut-off frequency of the filter should prevent aliasing effect but it should not filter out signals in the frequency band of interest. AA filters for voltage and current signals should be identical to prevent large energy errors at lower power factors [10]. For PMUs, the phase of the AA filter adds to the error of the instrument and needs to be characterized and corrected. When digital filters are used with oversampling ADCs, the group delay of these filters is a factor for measuring absolute phase in PMUs [11], [12].

\section{Gain Stage}

For some instruments, the gain stage may be not mandatory, as long as the signals from the transducer are within the ADC input range. Nevertheless, for a more flexible design, a differential input programmable gain amplifier (PGA) is desirable. This PGA will allow changing transducers in the future, without the need for re-designing the rest of the system. The PGA gain should be selected in such a way that the signals never exceed the ADC input range.
The gain stage can also act as an impedance buffer preventing loading of the transducer by the ADC input impedance (if this is insufficiently high). This is particularly important for shunts and RVD transducers where a relatively low impedance will load the transducer and give a predicable and correctable error.

The following additional factors may need to be considered:

- The gain stage may also incorporate an integrator for Rogowski coils.

- The temperature coefficient of gain setting resistors is an important factor for instrumentation.

- DC offsets in amplifiers are important for dccoupled systems and when integrators are used.

- The bandwidth of the amplifier must be considered as must the time constant.

- The common mode rejection ratio (CMRR) is important particularly for current measurements where one side of the circuit is not at earth potential.

- Noise is also a factor and the desired effective number of bits (ENOB) should be considered [13].

\section{D. $A D C$}

Accurate measurement of electrical quantities in the power grid is critical for monitoring the operating state of the grid and real-time decision making, because these measurements are the inputs for protection algorithms and control applications. The ADC, as part of the measurement chain in the analogue front end, plays an important role on the accuracy of a measuring instrument. There are a number of ADCs on the market which differ in terms of the architecture, resolution, sampling rate, conversion time, and number of channels.

One important characteristic that has to be taken in consideration is the way the ADC samples the input signal, i.e. the architecture. Successive approximation register (SAR) ADCs (Fig. 2) perform conversions by taking a sample from the input signal and achieving the conversion. This architecture provides very low latency. The latency depends mainly on the SAR algorithm processing time which is required to determine all the bits of the input signal; this can be reduced by operating the ADC at higher speed.

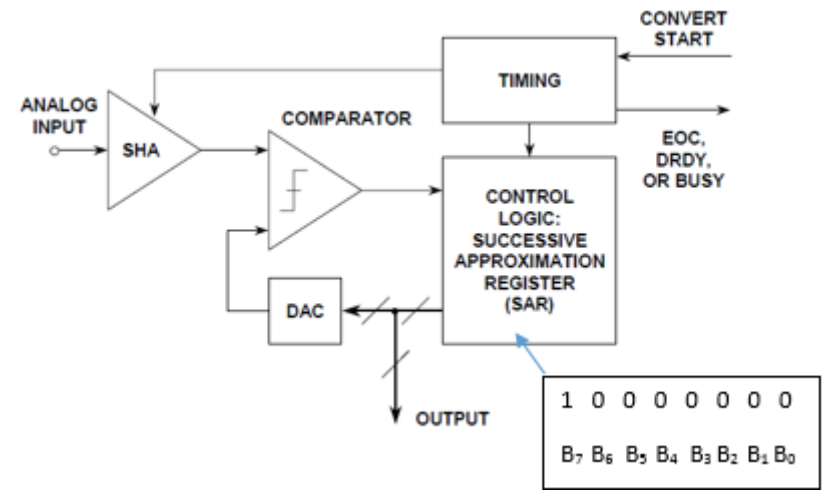

Figure 2. SAR ADC architecture (part of this figure is taken from [14]). 
For those applications requiring low latency and precise information in time when the sampling occurs, the SAR architecture is advantageous.

Sigma-delta (sometimes referred to as $\Sigma \Delta$ ) ADCs (Fig. 3.) sample the signal continuously for a specified time interval and output the conversion result that corresponds to the average of samples over that period of time. An important feature of this architecture is the oversampling capability and the digital filter, which aims to reduce the quantization noise generated internally in the ADC and, consequently, improve the signal to noise ratio (SNR) and ENOB.

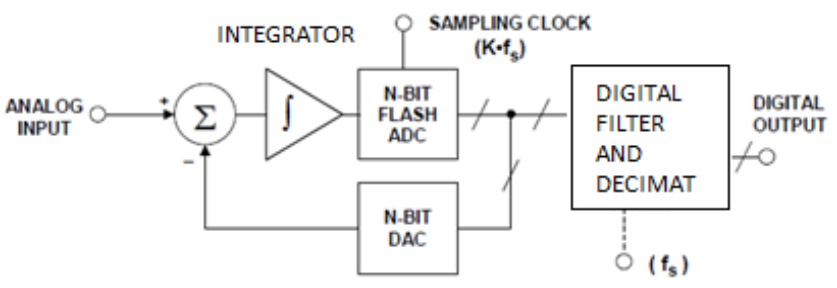

Figure 3. Sigma-delta ADC architecture [14].

Choosing the appropriate ADC therefore involves a tradeoff, but in most the cases the application itself guides the selection. That is, in applications where a very large bandwidth is required, the high speed architectures such as 'flash converters' are an appropriate choice, but the overall resolution is relatively low at 8 bits [14]. In industrial measurement applications including power, energy, and phasor measurements, where the bandwidth of interest is few $\mathrm{kHz}$, low speed architectures such as sigma-delta converters are advantageous because of the high resolution that this architecture offers. For power quality monitoring applications where the electromagnetic phenomena vary from a few $\mathrm{Hz}$ to $5 \mathrm{MHz}$ [15], medium speed architectures such as SAR converters are a better choice due to a good combination of speed and resolution.

Power system measurements require high-performance ADCs, with some desirable features such as: high resolution (typically 24-bit), differential inputs, simultaneous sampling and oversampling capabilities. The sigma-delta ADC architecture offers a better trade-off between resolution and sampling rate compared to a SAR ADC. Additionally, the delays introduced by delta-sigma ADCs can be calculated and therefore can be compensated in time-sensitive applications [12].

The higher the resolution of $\mathrm{ADC}$, the more data that is generated. A 24-bit device generates three 8-bit words per sample increasing the real-time burden of the digital signal processor (DSP), particularly for higher sampling rate systems. The improved noise performance of high resolution devices can be usefully employed by disregarding data bits of low significance to keep the processing manageable.

Over-range flags, where applicable, should be used to indicate to the CPU that saturation has occurred so that the user display can indicate that the result is invalid.

The ADC on some multichannel instruments can be multiplexed. The effect of this on inter-channel phase needs careful consideration because each channel may not be sampled at the same instant.

\section{E. Processing Unit}

The processing unit is typically a microprocessor, DSP, or field-programmable gate array (FPGA), which is responsible for performing the mathematical computation of electrical quantities from the digitized inputs. The processing unit should be fast enough and computationally capable to meet the application requirements. For example, for a phasor measurement unit (PMU) device, a "hard" real-time processing platform may be desirable.

The CPU must be fast enough to handle the data stream in real-time. Buffers can be used to hold data whilst the processor multitasks, but the processing speed must be sufficient to prevent the buffers overflowing and losing samples. The data can be indexed in some way (using a counter) to check for missed data which, if undetected, can give rise to significant errors.

There are a number of hardware factors that influence the performance of the processing unit [16], including: clock frequency, processor latency, memory access time, and the protocol used by the processor to communicate with peripheral devices. Software factors include: algorithm complexity and the ability to parallelize the tasks in order to process the information simultaneously in the cores of a multicore system processor. All of these factors need a careful consideration when deciding which processor is best for a particular application.

\section{F. Energy Metering Integrated Circuits}

Energy metering integrated circuits (IC) are specialized mixed-signal circuits, which may incorporate analogue frontend components, such as PGA and ADC, as well as processing unit. These circuits are convenient to ease the design of electricity meters and power quality monitoring instruments, as the embedded processor performs the necessary mathematical calculations. Nonetheless, it is important to verify the IC compliance with applicable standards, and also to verify the formulas implemented to calculate electrical quantities.

The internal composition of the aforementioned ICs varies among vendors, and among different models offered by a single vendor. A simplified common architecture is showed in Fig 4.

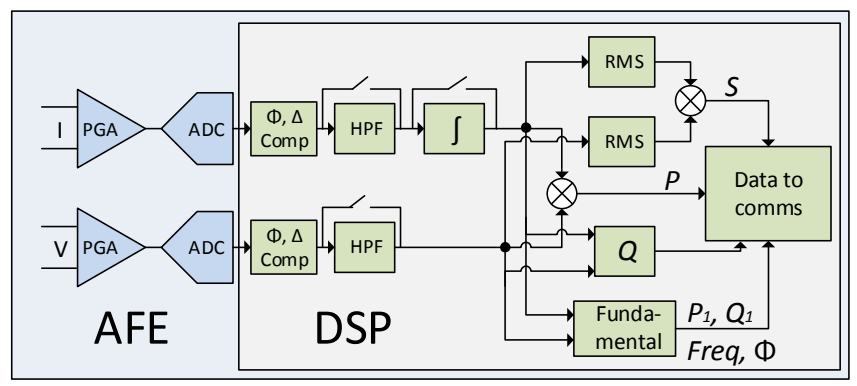

Fig. 4. Energy Metering integrated circuit block diagram. 
TABLE I. COMPARISON OF ENERGY METERING ICS FROM DIFFERENT VENDORS

\begin{tabular}{|c|c|c|c|c|c|c|c|}
\hline $\begin{array}{c}\text { Energy } \\
\text { Metering IC }\end{array}$ & Type & $\begin{array}{c}\text { ADC } \\
\text { Resolution }\end{array}$ & $\begin{array}{c}\text { Accuracy } \\
\text { (Active Power) }\end{array}$ & Bandwidth & $\begin{array}{c}\text { Supported } \\
\text { Current } \\
\text { Sensor } \\
\end{array}$ & $\begin{array}{l}\text { Supported } \\
\text { Standards }\end{array}$ & $\begin{array}{l}\text { Special Features/ } \\
\text { Comments }\end{array}$ \\
\hline ADE7878 & 3-Phase & 24-bit & $0.1 \%$ & $4 \mathrm{kHz}$ & CT, Rogowski & $\begin{array}{c}\text { EN 50470-3, } \\
\text { IEC 62053-21,22,23 }\end{array}$ & $\begin{array}{l}\text { Waveform } \\
\text { Sampling }\end{array}$ \\
\hline ADE9000 & 3-Phase & 24-bit & $0.1 \%$ & $7.2 \mathrm{kHz}$ & CT, Rogowski & $\begin{array}{c}\text { EN50470-3; } \\
\text { IEC 62053-21,22,23 } \\
\text { OIML R46; } \\
\text { ANSI C12.20 } \\
\text { IEC 62053-24 } \\
\end{array}$ & $\begin{array}{l}\text { Waveform Buffer } \\
\text { RMS } 1 / 2 \text { cycle }\end{array}$ \\
\hline ADE9153 & 1-Phase & 24-bit & $0.1 \%$ & $1.6 \mathrm{kHz}$ & CT, Shunt & $\begin{array}{c}\text { EN50470-3; IEC } \\
\text { 62053-21,22,23 } \\
\text { OIML R46; } \\
\text { ANSI C12.20 } \\
\text { IEC 62053-24 }\end{array}$ & Autocalibration \\
\hline 71M6513 & 3-Phase & 21-bit & $0.1 \%$ & $\begin{array}{l}\text { Estimated } \\
\text { at } 1.2 \mathrm{kHz}\end{array}$ & $\begin{array}{l}\text { CT, Rogowski, } \\
\text { Shunt }\end{array}$ & $\begin{array}{c}\text { IEC 62053, } \\
\text { ANSIC } 12.20 \\
\end{array}$ & Multiplexed ADC \\
\hline ATM90E36A & 3-Phase & 16-bit & $0.1 \%$ & $2 \mathrm{kHz}$ & CT, Rogowski & $\begin{array}{l}\text { IEC62052-11, } \\
\text { IEC62053-22 } \\
\text { IEC62053-23, } \\
\text { ANSI C12.1 and } \\
\text { ANSI C12.20 }\end{array}$ & $\begin{array}{l}\text { Lowest } \\
\text { temperature drift }\end{array}$ \\
\hline ATM90E26 & 1-Phase & 16-bit & $0.1 \%$ & $1.1 \mathrm{kHz}$ & CT, Shunt & $\begin{array}{l}\text { IEC62052-11, } \\
\text { IEC62053-21,23 }\end{array}$ & $\begin{array}{l}\text { Single-point } \\
\text { calibration for } \\
\text { active energy }\end{array}$ \\
\hline STPM32 & 3-Phase & 24-bit & $0.1 \%$ & $3.6 \mathrm{kHz}$ & $\begin{array}{l}\text { CT, Rogowski, } \\
\text { Shunt }\end{array}$ & $\begin{array}{l}\text { EN 50470-x, } \\
\text { IEC 62053-2x, } \\
\text { ANSI12.2x }\end{array}$ & $\begin{array}{l}\text { Dual mode } \\
\text { apparent energy } \\
\text { calculation }\end{array}$ \\
\hline
\end{tabular}

Some desirable features, from the metrology perspective, are the capability of compensating (via software), parameters such as phase, gain, and offset, as well as the IC current sensor compatibility. In Table I, a comparison of energy metering ICs features from different vendors is presented. It is worth noting that all IC models in Table I claim an accuracy of $0.1 \%$, despite their resolution, but only few models meet the requirements of the most constraining standards and international recommendations for tests and test conditions such as the OIMLR R46. The IEC 62053-24 standard for reactive energy at fundamental frequency, and the implementation of RMS measurements over half cycle, as per described in IEC 61000-4-30 standard, is also performed by some of these commercial devices.

Another important consideration when choosing an energy metering IC is the possibility to obtain the calculations of parameters related to the fundamental frequency, such as fundamental active power $\left(\mathrm{P}_{1}\right)$, fundamental reactive Power $\left(\mathrm{Q}_{1}\right)$, fundamental active energy and fundamental reactive energy. Such capabilities of distinguishing fundamental and nonfundamental powers and energies, as well as the possibility of updating (or upgrading) the IC firmware will provide the final design with some extra flexibility, as the standards and the test conditions are prone to change over the instrument's lifetime span.

\section{G. Time Stamp and Synchronization}

A time stamp is often required to keep track of sampled data. This time stamp can be readily achieved by means of a local (or remote) real-time clock (RTC) for many applications. However, some applications, such as PMUs, require an accurate time stamp source, with accuracy typically of 1 microsecond, such as from a global navigation satellite system (GNSS) clock. In some applications, it is desirable to control the timing of the analogue sampling to align with the clock source.

Clock stability and clock jitter are two important parameters for a clock, as they may have a significant impact on real-time applications. Small changes of the frequency from its default value could cause significant deviations of the clock from the actual time, whereas large amount of jitter could produce erroneous data and synchronization problems.

\section{SignAl PROCESSING}

Once the data has been converted to a digital representation, it should be processed accordingly to accurately calculate the required electrical quantities. Different approaches and formulas may be used for this purpose. This section reviews some of these formulas and techniques, informed by the latest issued applicable standards.

\section{A. Definitions for the Measurement of Electric Power Quantities}

To calculate electrical quantities such as energy, power (active and reactive), power factor, and frequency, different approaches may be used (e.g. Budeanu or Fryze definitions), which are equivalent in sinusoidal conditions, but lead to different and erroneous results when distorted or unbalanced situations exist [17]. The disadvantages of implementing definitions which are non-physical-based, such the Budeanu approach has been widely discussed and criticized by many authors [18]-[20], yet is often implemented in commercial 
metering instruments, as standards and regulatory bodies presently permit this approach [17].

The IEEE 1459-2010 standard [21], on the other hand, includes suitable definitions to perform the calculations of electrical measurements under different power quality conditions, such as sinusoidal, nonsinusoidal, balanced or unbalanced conditions, which are frequently present in realworld scenarios.

IEEE 1459-2010 standard is meant to be the reference for new electrical measuring instruments designs, particularly, for revenue purposes. The definitions of nonfundamental, nonactive power $S_{N}$ and its components, current distortion power $\left(D_{I}\right)$ and voltage distortion power $\left(D_{V}\right)$, "quantifies the overall amount of harmonic pollution delivered or absorbed by a load" and may be implemented by a new generation of electricity meters. Even though this standard was issued nearly ten years ago, it is very hard (if not impossible) to find commercially available measuring instruments implementing such electrical definitions.

\section{B. Phasor Measurement Units}

PMU applications require special features such as a precisely synchronized time reference for analogue sampling. It is also important to consider the computational capabilities of the processor unit and the phase delay introduced by the analogue front end, as well as the latency introduced by the digital filter of the sigma-delta ADC. For real-time control and protection applications, PMUs must also generate and output synchrophasor reports, typically using the standardized IEEE C37.118.2 [22] communications format, in a timely manner, and no later than the standardized reporting latency limits.

\section{CONCLUSION}

The design of a particular electrical measuring instrument can be achieved in many different ways, adopting a particular set of components, signal processing techniques, and formulas. With a large number of sophisticated hardware components available on the market, it can be difficult to select the most appropriate components for the target application. In this paper, a review of the advantages of some of these components and techniques has been given, to provide practical guidance for developing power system measurement devices.

\section{ACKNOWLEDGEMENT}

Quijano Cetina R. wishes to express his gratitude to the Mexican Energy Ministry (SENER) and the National Council for Science and Technology (CONACYT) for financing this study through the Scholarship CONACYTSECRETARIA DE ENERGIA- SUSTENTABILIDAD ENERGETICA 2016- ref.: 291041/ 439171.

This work has received funding from the European Union's Horizon 2020 research and innovation programme MEAN4SG under the Marie Skłodowska-Curie grant agreement 676042 .

\section{REFERENCES}

[1] G. Rietveld, J. P. Braun, P. S. Wright, P. Clarkson, and N. Zisky, "Smart grid metrology to support reliable electricity supply," 29th Conference on Precision Electromagnetic Measurements (CPEM 2014). pp. 680-681, 2014.

[2] JCGM, "Evaluation of measurement data-Guide to the expression of uncertainty in measurement," 2008

[3] IEC, "EN 61869-2:2012 Instrument transformers, Part 2: Additional requirements for current transformers." The International Electrotechnical Commission, 2012.

[4] IEC, "EN 61869-3:2011 Instrument transformers, Part 3: Additional requirements for inductive voltage transformers." The International Electrotechnical Commission, 2011.

[5] L. Peretto and R. Tinarelli, Sensors for PMUs. Elsevier Inc., 2016.

[6] N. G. Paulter, "Method for Measuring the Phase Spectrum of the Output of a Frequency Source Used in the Calibration of an Electroshock Weapon Characterization System," vol. 122, no. 35, 2017.

[7] F. Leferink, C. Keyer, and A. Melentjev, "Static energy meter errors caused by conducted electromagnetic interference," IEEE Electromagnetic Compatibility Magazine, vol. 5, no. 4. pp. 49-55, 2016.

[8] Y. Chen, Q. Huang, and A. H. Khawaja, "Interference-rejecting current measurement method with tunnel magnetoresistive magnetic sensor array," IET Sci. Meas. Technol., vol. 12, no. 6, pp. 733-738, 2018.

[9] Y. C. Chen, W. H. Hsu, S. H. Cheng, and Y. T. Cheng, "Antielectromagnetic interference and calibration schemes of flexible current sensor tag for reliable current detection in household appliance," in 2013 Transducers \& Eurosensors XXVII: The 17th International Conference on Solid-State Sensors, Actuators and Microsystems (TRANSDUCERS \& EUROSENSORS XXVII), 2013, pp. 1356-1359

[10] Analog Devices Inc, “ADE7878 Evaluation Board User Guide UG146." 2010.

[11] F. Hohn, J. Wang, and L. Nordström, "Design of a Distributed Signal Processing Unit for Transmission Line Protection in a Centralized Substation Protection Architecture," in IECON 2018 - 44th Annual Conference of the IEEE Industrial Electronics Society, 2018, pp. $138-144$.

[12] B. Pisani, "Accounting for delay from multiple sources in delta-sigma ADCs."

[13] A. J. Roscoe, S. M. Blair, B. Dickerson, and G. Rietveld, "Dealing With Front-End White Noise on Differentiated Measurements Such as Frequency and ROCOF in Power Systems," IEEE Trans. Instrum. Meas., vol. 67, no. 11, pp. 2579-2591, 2018.

[14] B. James and K. Walt, "Data Converter Architectures," in Data Conversion Handbook, Analog Devices, 2005, pp. 965-1022.

[15] "IEEE Recommended Practice for Monitoring Electric Power Quality," IEEE Std 1159-2009 (Revision of IEEE Std 1159-1995). pp. c1-81, 2009.

[16] M. Jiménez, R. Palomera, and I. Couvertier, Introduction to embedded systems: Using microcontrollers and the MSP430. 2014.

[17] R. Quijano Cetina, A. J. Roscoe, and P. S. Wright, "Challenges for Smart Electricity Meters due to Dynamic Power Quality Conditions of the Grid: A Review," 2017 IEEE Int. Work. Appl. Meas. Power Syst., pp. 1-6, Sep. 2017.

[18] D. Gallo, C. Landi, N. Pasquino, and N. Polese, "A New Methodological Approach to Quality Assurance of Energy Meters Under Non-Sinusoidal Conditions," 2006 IEEE Instrumentation and Measurement Technology Conference Proceedings. pp. 1626-1631, 2006.

[19] L. S. Czarnecki, "What is wrong with the Budeanu concept of reactive and distortion power and why it should be abandoned," IEEE Trans. Instrum. Meas., vol. IM-36, no. 3, pp. 834-837, 1987.

[20] A. J. Berrisford, "Metering and Harmonics - The Inequity Issue A Discussion Presentation." 2016. 
[21] "IEEE Standard Definitions for the Measurement of Electric Power Quantities Under Sinusoidal, Nonsinusoidal, Balanced, or Unbalanced Conditions," IEEE Std 1459-2010 (Revision of IEEE Std 1459-2000). pp. 1-50, 2010.

[22] "IEEE Standard for Synchrophasor Data Transfer for Power Systems," IEEE Std C37.118.2-2011 (Revision of IEEE Std C37.1182005). pp. 1-53, 2011. 